\title{
PROPOSIÇÃO DE UM NOVO MÉTODO DE GERENCIAMENTO DE REQUISITOS NA ANÁLISE DE NEGÓCIOS
}

\section{PROPOSITION OF A NEW CUSTOMER REQUIREMENT MANAGEMENT METHOD FOR BUSINESS ANALYSES}

\author{
Paula Bartholomay Kothe* E-mail: paulabkothe@gmail.com \\ Ângela Maria Marx* E-mail: angela.marx@ufrgs.br \\ Alejandro Germán Frank* E-mail: frank@producao.ufrgs.br \\ *Universidade Federal do Rio Grande do Sul (UFRGS), Porto Alegre, RS
}

Resumo: O presente estudo tem como objetivo propor um modelo que integra e consolida diferentes modelos existentes na literatura para o processo de Gerenciamento de Requisitos, visando um horizonte abrangente que considere os detalhes das etapas que devem ser gerenciadas. Para tanto, foram revisados cinco modelos, dois da área de software, dois da área de produtos e um da área de análise de negócios. A partir do comparativo destes modelos, mediante o uso de conceitos de análise de conteúdo, obteve-se como resultado um modelo integrado que contempla cinco fases e 16 etapas que visam assegurar uma melhor performance do processo com base no atendimento das necessidades dos clientes. Após a obtenção do modelo integrado, o mesmo foi utilizado em um caso prático visando avaliar sua utilidade para a realização de diagnósticos da gestão por requisitos nas empresas. A principal contribuição se resume em um modelo completo que preenche as lacunas dos modelos existentes, aplicável a diversos setores, não se limitando ao desenvolvimento de softwares e produtos tradicionais deste mercado.

Palavras-chave: Gestão de Requisitos. Análise de Negócios.

Abstract: This article aims to propose a model that integrates and consolidates different models existing in the academic literature that describe the process of Requirements Management, aiming to obtain a broad vision considering all the details of the steps that should be managed. To obtain this result, five models, two in the software area, two in the products area and one business analysis area were reviewed. From the comparison of these models, through a content analysis, it have been obtained as a result an integrated model which includes five phases and 16 steps ensuring a better process performance regarding customer's needs. After obtaining the integrated model, it was used in a case study to evaluate its utility regarding diagnostic management performances requirements on companies. The most important contribution is a complete model that fill gaps in those ones that already exist and applicable to multiple sectors, not limited to the development of software and traditional product of this market.

Keywords: Requirement Management. Business Analysis.

\section{INTRODUÇÃO}

O mercado oriundo do desenvolvimento de softwares de gestão vem alcançando parcelas de mercado em um ritmo acelerado, devido à dependência das 
empresas por sistemas que auxiliem o gerenciamento de processos complexos, a fim de orientar a sua tomada de decisão (CORDEIRO, 2010; THONG et al., 2011). Seguindo esta lógica, a utilização de técnicas e conceitos de Gerenciamento de Requisitos (GR) tornou-se uma prática fundamental para a obtenção do sucesso nestes projetos. Sob este contexto, um requisito pode ser definido como uma necessidade que deve ser atingida, definida por condições mensuráveis e limitado por restrições (PARVIAINEN; TIHIENEN; VAN SOLINGEN, 2005). Para Hazan (2003), o GR tem como objetivo principal controlar a evolução dos requisitos, seja por constatação de novas necessidades ou por deficiências nos requisitos já registrados até o momento.

De acordo com Koscianski (2006), a qualidade de um software depende essencialmente dos requisitos. Não-conformidades, erros lógicos, conceituais e omissões na especificação de requisitos podem ser propagados por todo o projeto, resultando para a empresa prejuízos diversos, que oscilam de acordo com as necessidades de utilização do sistema desenvolvido, sua aplicabilidade e riscos que o seu uso podem ocasionar (SALES, 2011).

Uma pesquisa realizada pela Standish CHAOS Chronicles (2003) com empresas americanas aponta que apenas $28 \%$ dos projetos de software conseguem ser finalizados no tempo especificado e dentro do orçamento e que, além disso, apenas $52 \%$ dos projetos concluídos apresentam a funcionalidade proposta incialmente. Ainda, de acordo com esta pesquisa, aproximadamente $40 \%$ dos custos de projetos estão relacionados a retrabalhos. Sob esta mesma análise, o uso de requisitos incompletos, ambíguos ou esquecidos foi levantado como uma das principais causas que levam os projetos a extravasarem o seu planejamento. Tais indicadores comprovam o contexto em que se encontram as empresas de desenvolvimento de software quanto à realização de seus projetos, impactando diretamente no valor percebido pelos clientes e influenciando negativamente na imagem da empresa. Apesar de o conceito de GR ter se originado no ramo de software, este cenário não é restrito a ele, podendo ser aplicável em qualquer empresa que lide com requisitos e atendimento das necessidades de seus clientes (BABOK, 2009; THONG et al., 2011). 
Para Bruce e Cooper (2000), as empresas poderiam ser mais bem sucedidas se fosse dada maior atenção ao processo de captura e documentação dos requisitos nas fases iniciais do desenvolvimento de produtos e sistemas, visto que o custo de correção dos defeitos tende a aumentar em uma proporção de 10 vezes a cada etapa realizada (SOMMERVILLE, 2007; MYERS, 1979). Justifica-se tal orientação pelo fato de que um bom desempenho inicial resulta em redução de alocação de recursos físicos do projeto, além de garantir a qualidade dos documentos que servirão para a construção do novo produto (SOMMERVILLE, 2007; MYERS, 1979).

Sob este contexto se destaca a importância do setor de análise de negócios nas empresas, uma vez que esta área atua como facilitadora, comunicadora e solucionadora de problemas na gestão do escopo da solução proposta, descobrindo, analisando e gerenciando os requisitos levantados (IIBA, 2011). Portanto, o principal ponto de partida para a redução de falhas e retrabalhos nos processos de desenvolvimento se concentra na utilização dos conceitos de análise de negócios das empresas.

Motivado por uma problemática real e de origem prática, este trabalho propõe auxiliar empresas no processo de Gerenciamento de Requisitos, minimizando tanto problemas de prazo e custo como também de qualidade percebida pelos clientes. Para tanto, este estudo busca apresentar uma compilação de diversos modelos de $\mathrm{GR}$, explorando as lacunas encontradas de forma que a sua aplicabilidade seja também válida para outros segmentos que não somente os de software, já bastante explorados pela literatura. Tal proposição foi investigado um caso prático de uma empresa de consultoria com foco na gestão da cadeia de suprimentos e demanda.

Este artigo é composto por cinco seções, sendo a primeira delas a introdução, que contextualiza o tema, sua problemática e justifica o desenvolvimento do estudo. A segunda seção apresenta o referencial teórico, o qual apresenta uma revisão bibliográfica referente ao processo de desenvolvimento de software, análise de negócios e gestão de requisitos, com a finalidade de sustentar o tema desenvolvido e na terceira seção destacam-se os procedimentos metodológicos utilizados. Os resultados obtidos serão discutidos na seção quatro e, por fim, na última seção são apresentadas as considerações finais, seguida da lista de referências bibliográficas utilizadas no trabalho desenvolvido. 


\section{REFERENCIAL TEÓRICO}

Para compreensão e embasamento do estudo, a seguir é apresentada uma breve revisão teórica sobre os tópicos que orientaram esta pesquisa, englobando conceituação e caracterização do processo de desenvolvimento de software, a conceituação de requisitos e o seu gerenciamento e, por fim, análise de negócios.

\subsection{Processo de desenvolvimento software}

Um processo de desenvolvimento de software define o conjunto de atividades que serão conduzidas no contexto do projeto, os recursos necessários, os artefatos e os procedimentos a serem adotados na realização de cada uma das atividades (GRUHN, 2002; FALBO, 1998). Segundo Sommerville (2007), dentre as várias atividades associadas, de acordo com o modelo clássico de desenvolvimento, existem as etapas de definição de requisitos, projeto de software, implementação e teste de unidades, integração e teste do sistema e, por fim, operação e manutenção.

O modelo de um processo de desenvolvimento de software é uma representação abstrata, onde são apresentadas informações específicas do processo a partir de uma perspectiva particular e, desta forma, processos bem estruturados são fundamentais para se obter produtos de qualidade (SOMMERVILLE, 2004). Para Pressman (1995), essa qualidade pode ser definida como a conformidade a requisitos funcionais e de desempenho explicitamente declarados, a padrões de desenvolvimento claramente documentados e a características implícitas que são esperadas de todo software profissionalmente desenvolvido. Porém, apesar do aumento da sua importância e necessidade para competitividade empresarial perante o mercado, a realidade vivenciada pelas empresas desenvolvedoras de software ainda é desafiadora (THONG et al., 2011).

No processo de desenvolvimento é geralmente nas etapas iniciais que ocorrem os maiores problemas, visto que são àqueles de maior impacto negativo para correção nas fases futuras. Estas etapas constituem o processo de Engenharia de Requisitos, que tem sido reconhecida como uma das mais importantes fases do processo de desenvolvimento de software (KOTONYA; SOMMERVILLE, 1998). A 
identificação do que construir pode ser considerada como a parte mais árdua na construção de um software, visto que nenhuma outra compromete tanto o trabalho se elaborada de forma incorreta, bem como não há outra parte ofereça comparado grau de dificuldade para efetuar correções posteriores (BROOK, 1986; SOMMERVILLE, 2007).

\subsection{Gestão de requisitos}

A área em que a problemática de requisitos tem sido estudada com profundidade é a de Engenharia de Requisitos, que é a primeira fase da Engenharia de Software, em que são contempladas as etapas de elicitação, análise-priorização, documentação e verificação de requisitos ao longo de todo o projeto (KOTONYA, SOMMERVILLE, 2000; SOM-MERVILLE, 2007). Além disso, devido à grande instabilidade dos requisitos ao longo de um processo devido às mudanças no sistema e problemas de entendimento por parte dos usuários, foi criado o processo denominado de Gestão de Requisitos, que trata da gestão da mudança e da rastreabilidade dos requisitos durante seu projeto (PARVIAINEN; TIHINEN; SOLINGEN, 2005; SOMMERVILLE, 2007). Em alguns casos, a Gestão de Requisitos é considerada um processo paralelo às etapas anteriores do processo de desenvolvimento de software e voltada para o gerenciamento de mudanças (ALVES, 2001; PARVIAINEN; TIHINEN; SOLINGEN, 2005; SOMMERVILLE, 2007). Destacase que, neste trabalho, o conceito de gerenciamento de requisitos é utilizado como sinônimo da Engenharia de Requisitos, contemplando também o processo de gerenciamento de mudanças.

Requisitos incluem desejos coletivos, perspectivas e expectativas dos vários clientes que constituem a principal fonte de informação para o empreendimento construtivo, ou seja, correspondem a funções, atributos e demais características do produto ou serviço requerido (KAMARA et al., 2002). Requisitos de boa qualidade devem ser necessários, inteligíveis, exequíveis, exatos, completos, testáveis, rastreáveis, exclusivos e não devem exigir soluções prematuras. Estas características são importantes, pois uma vez atendidas, garantem a qualidade da informação que irá entrar no projeto e gerar valor ao produto ou serviço (KOTONYA; 
SOMMERVILLE, 2000; YOUNG, 2003). Porém, para a análise de negócios, o uso do conceito de requisitos deve ser tomado a partir de um sentido mais amplo. De acordo com o IIBA (2011) os requisitos incluem, mas não estão limitados a, condições ou capacidades futuras ou passadas em um empreendimento e descrições de estruturas organizacionais, papéis, processos, políticas, regras e sistemas de informações. Um requisito pode descrever o estado presente ou futuro de qualquer aspecto do empreendimento.

O entendimento superficial dos requisitos do cliente gera um conhecimento impreciso que afeta negativamente o projeto e a produção do produto, em termos de qualidade, tempo despendido e custo (JIAO; CHEN, 2006). Neste caso, a atenção dada aos requisitos desde o início do projeto tem como objetivo assegurar a realização das atividades necessárias para incorporar as necessidades dos clientes aos produtos desenvolvidos pela empresa, reduzindo assim os retrabalhos do processo (MARTINI, 2003; MARX, 2009). Além disso, torna-se necessário captar as necessidades e expectativas não explicitadas do cliente final para que essas informações forneçam subsídios para os tomadores de decisão na concepção e no projeto do produto. A falta desse conhecimento pode comprometer o resultado final do produto, resultando em trade-offs nos quais predomina a visão dos técnicos no processo (ULRICH; EPPINGER, 2000).

Existem atividades dentro do desenvolvimento de software que estão relacionadas diretamente aos requisitos. Sob este contexto, Sommerville (2000) discute a necessidade de manter uma GR em paralelo com o gerenciamento de mudanças. Mudanças no processo são inevitáveis e quanto mais eficaz for a gestão dos requisitos, maior também a probabilidade de sucesso do projeto (SUN et al, 2005; BRAY, 2002). Miron (2008) destaca que a boa GR contribui para o delineamento mais claro dos resultados que se espera de um projeto e facilita a identificação das habilidades e carências que tornam os envolvidos hábeis ou não a desenvolvê-lo. Para Martini et al. (2003) a GR tem como finalidade propiciar um esclarecimento das informações dos requisitos. Esta comunicação deve ser feita entre empresas, clientes e fornecedores, os quais tem como função, manter a clareza entre os resultados esperados pelos clientes, descrever o propósito do 
produto ou serviço, torná-los rastreáveis e ser um guia para todos os envolvidos com o projeto.

A literatura apresenta diferenciados modelos de referência para a GR, diferindo-se estes de acordo com suas abordagens e atividades. A Engenharia de Requisitos descrita por Kotonya e Sommerville (1998) é composta por quatro fases: (i) elicitação ou levantamento de requisitos; (ii) análise e negociação dos requisitos; (iii) documentação dos requisitos e (iv) validação de requisitos. A Gestão de Requisitos de Young (2003) é baseada na gestão de processos, a qual se baseia em processos que incluem atividades destinadas ao acompanhamento de tarefas, o que é uma abordagem diferenciada do modelo descrito anteriormente. Este autor apresenta um desdobramento das etapas básicas da engenharia de requisitos em diversas atividades, entre as quais está o entendimento das necessidades de clientes e usuários; a análise, especificação e priorização de requisitos; a alocação destes em subsistemas; o monitoramento e gerenciamento dos requisitos e a validação destes.

Para Rozenfeld et al. (2006) requisitos são características que o produto deve atender segundo os valores-meta, desdobrados a partir de requisitos do cliente, que são as necessidades organizadas, categorizadas e estruturadas. Apesar disso, no caso do desenvolvimento de produtos, a GR não é diretamente citada e estão dispersa no meio das etapas do processo de identificação das necessidades, análise e criação de valor e descontinuação ao final do seu ciclo de vida.

Seguindo esta lógica de requisitos em produtos, Marx e Paula (2009) destacaram a necessidade da elaboração de uma sistemática para o desenvolvimento de produtos sustentáveis (GRPS). Esta sistemática de GR proposta baseia-se em três etapas: (i) definição dos objetivos de sustentabilidade do negócio; (ii) definição dos requisitos do negócio e (iii) definição dos requisitos do sistema-produto sustentável. Dentre estas macro fases estão relacionadas diversas tarefas que visam assegurar que os produtos desenvolvidos reflitam 0 posicionamento quanto a sustentabilidade. Já a área de conhecimento descrita pelo BABOK (2009) relativa ao GR descreve as tarefas que devem ser executadas para garantir que todas as partes interessadas tenham um entendimento compartilhado 
da natureza de uma solução e para assegurar que essas, com autoridade de aprovação, estejam de acordo quanto aos requisitos que a solução deva atender.

\subsection{Análise de negócios}

O conceito de análise de negócios consiste no conjunto de atividades e técnicas utilizadas para servir como ligação entre as partes interessadas no intuito de compreender a estrutura, políticas e operações de uma organização e para recomendar soluções que permitam que a organização alcance suas metas. A análise de negócios representa a habilidade de analisar um problema por múltiplas perspectivas e considerar diversas possibilidades de solução com base em uma visão realística de custo versus benefício coerente com a visão estratégica da organização (IIBA, 2009; NEVES, 2012).

A análise de negócio é geralmente praticada no contexto de um projeto, onde o praticante atua como um intermediário entre os interesses das partes, consolidando os pontos de vista das partes interessadas do negócio e comunicando as suas necessidades para um provedor de solução. A natureza do trabalho nesta área envolve o levantamento e assimilação de grandes quantidades de informação e compreensão desses requisitos, o que é fundamental para o sucesso dos projetos desenvolvidos (TEMPLETON, 2012; PODESWA, 2008).

Seguindo esta lógica, a definição de requisitos é a tarefa-chave da análise de negócios. É essencial que os requisitos estejam completos, claros, corretos e consistentes, pois esses servem como pilares da solução para as necessidades do negócio. O analista de negócios deve compreender as técnicas comumente usadas para elicitar requisitos, ser capaz de selecionar as técnicas apropriadas para uma dada situação e ser conhecedor das tarefas para preparar, executar e completar cada técnica (IIBA, 2011). Com o intuito de auxiliar profissionais dessa área, em 2003 foi criado o IIBA (Internacional Institute of Business Analysis) e, dentro deste aspecto, foi elaborado o BABOK (Business Analysis Body of Knowledge). Este guia reúne uma série de práticas e atividades comumente aplicadas no ambiente da análise de negócios, além de apresentar e indicar uma série de técnicas e atividades a serem utilizadas em cada etapa da análise. 


\section{PROCEDIMENTOS METODOLÓGICOS}

Nessa seção inicialmente é apresentada uma breve caracterização da pesquisa aplicada neste estudo. Na sequência é descrito o desdobramento das etapas de trabalho propostas para o desenvolvimento de um método de GR aplicada à análise de negócios.

\subsection{Método de pesquisa}

O presente estudo classifica-se como uma pesquisa de natureza aplicada, visto que busca gerar conhecimento de aplicação prática, pois serão desenvolvidas novas contribuições para a solução de problemas relacionados ao gerenciamento de requisitos (BOAVENTURA, 2009). A abordagem do estudo é considerada qualitativa, já que busca analisar modelos e conceitos relacionados ao GR a partir de diferentes perspectivas. O objetivo do trabalho se caracteriza como explicativo, pois será desenvolvido um modelo, obtendo como contribuição uma estrutura formal que poderá ser utilizado por outras empresas em casos particulares. Os procedimentos são caracterizados em duas etapas: primeiramente é proposto um desenvolvimento conceitual de um método integrado baseado em uma comparação de modelos mediante análise de conteúdo. A segunda etapa consiste em um estudo de caso no qual se analisa a realidade de uma empresa à luz do modelo desenvolvido na primeira etapa.

\section{MÉTODO DE TRABALHO}

O método de trabalho foi dividido em duas partes, uma conceitual e outra prática que foram desdobradas em cinco etapas, sendo as quatro primeiras referentes à conceitual e a última à prática. As etapas detalhadas dividiram-se da seguinte maneira: (i) seleção dos modelos de GR na literatura (ii) mapeamento das atividades nos modelos selecionados; (iii) comparação entre os modelos; (iv) consolidação de um novo modelo e, por fim, (v) diagnóstico de uma empresa com relação ao modelo proposto. 


\subsection{Seleção de modelos de gestão de requisitos na literatura}

Existem diferentes modelos de GR na literatura apresentando diferentes abordagens e estruturas. Desta forma, a primeira fase do trabalho iniciou-se com a definição dos modelos que seriam utilizados como referencial para o estudo em questão. Esta escolha foi baseada em uma investigação dos modelos mais representativos na literatura, considerando como base da pesquisa livros e artigos recentemente publicados (2000 a 2014) a partir de palavras-chaves como análise de negócios, engenharia de software, engenharia e gerenciamento de requisitos. Após esta coleta de artigos, foram selecionados os modelos mais citados e filtrados aqueles que se enquadram na proposta deste trabalho.

\subsection{Mapeamento das atividades nos modelos selecionados}

$\mathrm{Na}$ segunda etapa do trabalho as atividades dos modelos escolhidos foram levantadas e recompostas com a finalidade de melhor expressar o seu significado dentro do contexto elaborado seguindo sempre uma ordem cronológica do processo. Além das atividades mapeadas foram encontradas ferramentas e técnicas que serviram de base para um melhor entendimento e comparação das atividades dos diferentes modelos de GR na próxima etapa.

\subsection{Comparação dos modelos de gestão de requisitos}

Na terceira etapa do estudo, para a comparação dos modelos selecionados foram utilizados conceitos da análise de conteúdo, técnica de investigação que tem por finalidade a descrição objetiva e sistemática do conteúdo do manifesto de comunicação (GODOY, 1995). A abordagem utilizada foi a proposta por Bardin (2004), que sugere três regras de codificação para transformar os dados coletadas em informações organizadas. Estas três regras são: (i) regra de recorte, na qual se escolhe a unidade de análise; (ii) regra de enumeração, que define a forma de contagem da unidade de análise; e (iii) regra de categorização, que define a forma de construção do agrupamento dos dados. A partir da regra de recorte, as atividades 
de gerenciamento de requisitos foram agrupadas por unidades de significado comuns no conteúdo analisado, ou seja, os modelos foram postos lado a lado e as suas atividades foram dispostas uma abaixo da outra e ao lado as atividades correspondentes de outros modelos selecionados. A regra de enumeração somente foi utilizada para considerar se uma atividade estava ou não no modelo proposto (contagem binária), não considerando a frequência das frases e palavras citadas. Todas estas regras foram construídas por meio de um processo de decisão entre os autores. Por fim, a regra de categorização foi aplicada na etapa quatro deste trabalho e é descrita posteriormente.

\subsection{Consolidação de um modelo de gestão de requisitos}

A quarta etapa do estudo englobou a criação de um modelo de GR baseada na análise comparativa de todos os modelos selecionados da literatura a partir da lista de atividades e das lacunas identificadas. A terceira etapa proposta na análise de conteúdo, regra de categorização, iniciou-se com a identificação das etapas de GR, classificação destas em grupos comuns, definição das fases e, então para a categorização foi utilizado o processo de definição dos nomes das categorias, construídas com base no critério semântico, o qual define a categoria conforme o significado geral dos elementos.

\subsection{Diagnóstico da empresa com relação ao modelo proposto}

$\mathrm{Na}$ última etapa do estudo a sistemática integrada na etapa quatro foi testada mediante o diagnóstico do processo de GR em uma empresa específica. A empresa escolhida foi uma consultoria com foco na gestão da cadeia de suprimentos e demanda que trabalha com a GR aplicada na análise de seus negócios. Esta foi escolhida pelo fato de já trabalhar com a metodologia de GR há 4 anos mas que, ainda assim, apresentava índices de retrabalhos inaceitáveis para o padrão de sua organização. Tal índice é medido pelo número de horas previstas para cada atividade em comparação ao gasto para sua realização. Para a coleta de dados com relação ao procedimento utilizado pela empresa escolhida foi empregado o método 
de triangulação de dados, fundamento lógico para se utilizar várias fontes de evidencias que permitem que o pesquisador dedique-se a várias fontes distintas de informação para o desenvolvimento de linhas convergentes de investigação (YIN, 2001). Foram realizadas entrevistas semiestruturadas com todas as áreas envolvidas com o processo de gestão de requisitos (área de consultoria, qualidade de software, análise de negócios, desenvolvimento e comitê do produto) com o objetivo de entender o processo atual de GR. A área de consultoria é a responsável pela implantação dos projetos nos clientes enquanto que a análise de negócios é quem faz a tradução das necessidades destes em funcionalidades. A equipe de desenvolvimento é a que projeta e elabora o produto e a área qualidade de software é quem faz os testes para validação das novas funcionalidades. Por fim, o comitê do produto é quem aprova ou não as necessidades traduzidas em novos produtos e serviços propostos pelos interessados.

Além disso, durante o processo de triangularização foi comparado o processo atual da empresa com o modelo proposto na literatura (Figura 2), bem como o nível de realização de cada uma das atividades. Para tanto foram realizadas reuniões para que todas as etapas pudessem ser analisadas e discutidas com todos os envolvidos. O resultado destas avaliações, junto à observação direta e à análise de documentos específicos (processos mapeados, documentos utilizados, manuais e treinamentos), gerou conhecimento necessário para a aplicação de um questionário em que todos os envolvidos deveriam preencher o grau de realização de cada uma das atividades (de 0 a 100\%) propostas de acordo com o seu processo atual.

\section{PROPOSIÇÃO DO MODELO CONCEITUAL INTEGRADO PARA GESTÃO DE REQUISITOS}

Os resultados deste trabalho obedecem a metodologia proposta de acordo com as etapas conceituais e práticas. Os resultados relativos ao modelo conceitual, foram divididos em três partes: definição dos modelos e mapeamento das atividades, a análise comparativa entre os modelos e apresentação do modelo final integrado para o gerenciamento de requisitos. Por fim, a etapa prática considera a 
avaliação do modelo em um caso aplicado, visando analisar a aplicabilidade da proposta a partir de um diagnóstico do processo utilizado pela empresa.

\subsection{Seleção dos modelos e mapeamento das atividades}

Como resultado da pesquisa realizada com os modelos mais representativos, foram selecionados dois modelos de gestão de requisitos para o desenvolvimento de softwares (KOTONYA, SOMMERVILLE, 2000; YOUNG, 2003), dois modelos focados no desenvolvimento de produtos (ROZENFELD, 2006; MARX e PAULA, 2011) e um modelo de gerenciamento de requisitos com foco na análise de negócios (BABOK, 2011). A evolução cronológica desses modelos também aponta a progressão das áreas de estudo dentro da gestão de requisitos, que começou focalizada especificamente na área de desenvolvimento de softwares, posteriormente passou à área de desenvolvimento de produtos e finalmente alcançou uma abrangência maior considerando a análise de negócios.

\subsection{Comparativo entre os modelos}

O desenvolvimento de um comparativo serviu para fornecer uma representação visual e por escrito das atividades e relações que são relevantes para a área de negócio, ajudando a observar os aspectos dos requisitos a partir de diferentes perspectivas. O quadro apresentado na Figura 1 apresenta a comparação entre os cinco modelos selecionados com a descrição das atividades de cada um. Os blocos, ressaltados em cor cinza, representam atividades que não foram consideradas de acordo com a abordagem utilizada.

As atividades presentes em cada modelo e similares aos outros são apresentadas lado a lado e foram agrupadas globalmente em cinco fases principais. Essas fases consistem conjuntos de atividades com objetivos comuns no processo de GR, sendo elas: (i) Elicitação, (ii) Especificação, (iii) Gerenciamento do Escopo, (iv) Validação, e (v) Monitoramento. A partir deste quadro comparativo é possível identificar lacunas presentes em cada um dos modelos, bem como a compreensão das atividades comuns e exclusivas de cada um. Algumas dessas atividades 
comuns podem apresentar diferentes nomenclaturas, dando origem a quadros de referências, e para tanto, na comparação foram buscadas sínteses coincidentes e divergentes de ideias como é apresentado na proposta de um modelo integrado de acordo com a Figura 2. A seguir, são discutidas cada uma das fases apresentadas no quadro comparativo. 


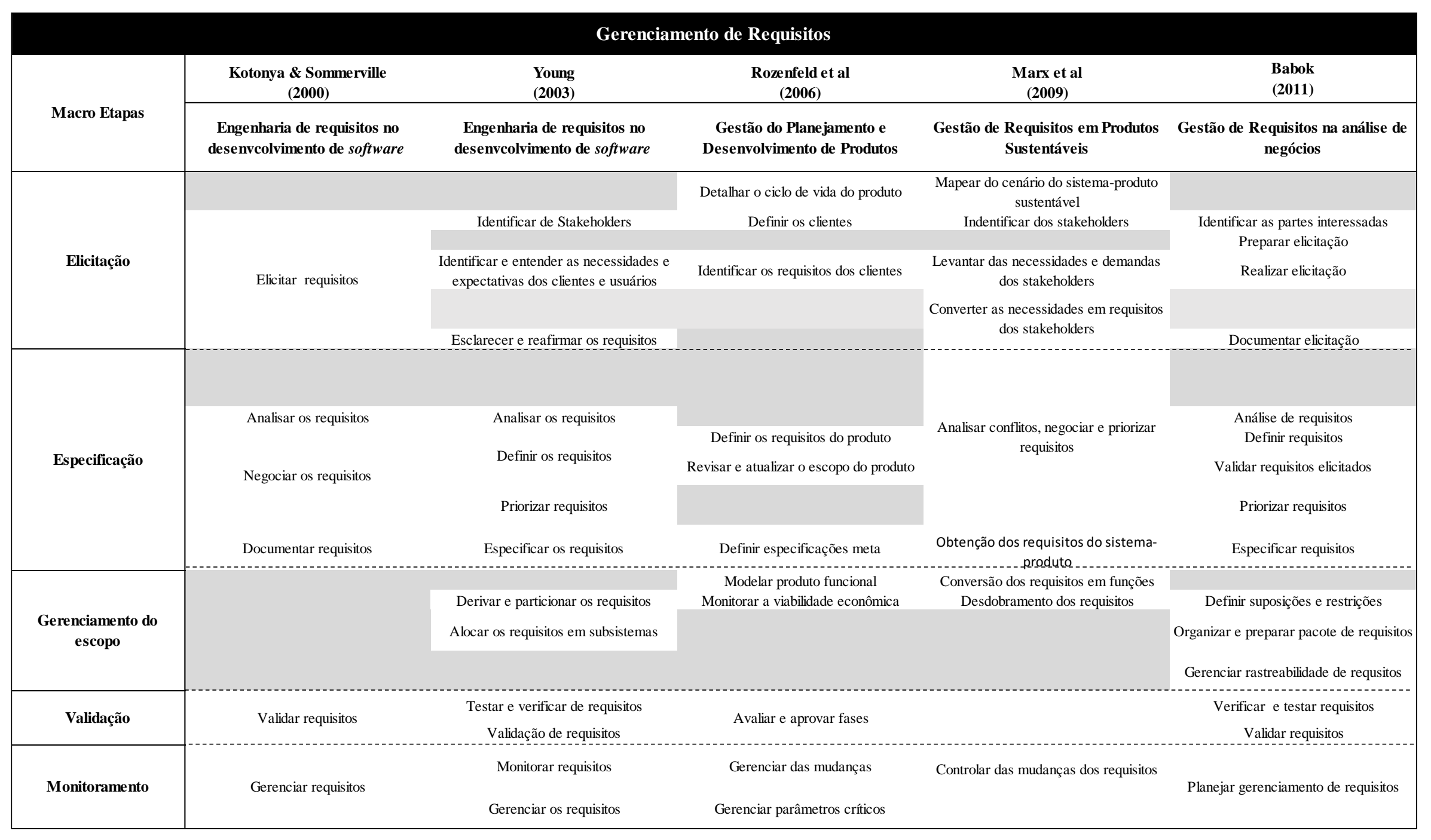

Revista Produção Online, Florianópolis, SC, v.15, n. 4, p. 1481-1510, out./dez. 2015. 


\subsubsection{Fase 1: Elicitação}

A primeira fase foi nomeada como "Elicitação" pelo fato de contemplar as atividades referentes aos primeiros contatos com a área requisitante, captando e entendendo as suas necessidades a partir do momento em que uma nova oportunidade é detectada. É nesta fase que são identificadas as expectativas e necessidades dos stakeholders com relação ao que deve ser desenvolvido. No comparativo entre os modelos, a primeira atividade referente a Gestão de Requisitos proposta por Rozenfeld (2006), se refere ao detalhamento do ciclo de vida do produto que, em outras palavras, equivale à atividade citada no modelo de Marx e Paula (2011), de mapeamento do cenário do sistema-produto, porém especificamente para produtos sustentáveis. Nos demais modelos estudados, esta etapa não é citada e o primeiro nível do processo de GR começa com outras atividades. Para Young (2003) e Babok (2011) o primeiro nível corresponde do processo de identificação de stakeholders, sendo que Babok (2011) ainda reforça a necessidade de se preparar para o processo elicitação antes da atividade ser realizada de fato.

Após a definição dos stakeholders, os autores prezam pela identificação das necessidades do cliente. Kotonya e Sommerville (2000) definem esta etapa como uma macro etapa de elicitação de requisitos enquanto outros autores já a descrevem com um maior detalhamento, considerando as etapas de preparação e condução da análise dos interessados, realização da elicitação e documentação do que foi definido.

Os modelos de Marx e Paula (2011) ainda considera a atividade de conversão das necessidades dos requisitos dos stakeholders para, posteriormente, documentar e dar a elicitação como validada, pois desta forma, os mesmos já terão sido captados, analisados e alinhados entre todos os stakeholders, eliminando possíveis desentendimentos entre as áreas envolvidas. 


\subsubsection{Fase 2: Especificação}

A segunda Fase, nomeada como "Especificação" é referente a atividade de documentação da especificação funcional ou técnica, que servirá de base para o desenvolvimento do produto ou serviço. Marx e Paula (2011) considera as atividades detalhadas por outros autores (i.e. análise de requisitos, definição de requisitos e validação dos requisitos) como uma única atividade. Além disso, ela ainda ressalta uma fase a mais nesta etapa quando comparado com os outros modelos, a análise de conflitos. Tal atividade tem como objetivo esclarecer as lacunas a partir de possíveis conflitos gerados entre os diferentes stakeholders, visto que cada interessado pode ter uma perspectiva particular a partir das experiências pessoais de cada um.

Todos os outros autores, exceto Rozenfeld (2006), citam direta ou indiretamente a análise dos requisitos como próxima etapa, momento em que se analisa os requisitos captados pelas partes interessadas, no intuito de permitir que a equipe do projeto implemente a solução que irá atender às necessidades da organização e das partes interessadas, avaliando o estado atual do negócio para identificar e recomendar melhorias. Após esta análise, os requisitos podem ser considerados como definidos. Posteriormente, Young (2003), Marx e Paula (2011) e Babok (2011) citam a atividade de priorização dos requisitos de forma específica, a qual representa o processo de decisão para determinar a importância relativa dos requisitos, que pode ser baseada no seu valor relativo, risco, dificuldade de implementação ou qualquer outro critério que se julgue necessário para o procedimento de gestão de requisitos a ser implementado.

Por fim, a etapa de Especificação é finalizada com o documento de especificação formal acabado e validado pelas áreas envolvidas (KOTONYA e SOMMERVILLE, 2000; YOUNG, 2003; ROZENFELD et al., 2006; BABOK, 2011). Isso representa um documento com os requisitos levantados, analisados e já convertidos na realidade em que serão desenvolvidos de acordo com um modelo estruturado que contenha todas as informações necessárias para que o produto possa ser desenvolvido. 


\subsubsection{Fase 3: Gerenciamento do escopo}

Todos os requisitos das soluções e das partes interessadas devem ser avaliados para assegurar que estejam dentro da necessidade declarada. Desta forma, a Fase 3 denominada como "Gerenciamento do escopo" engloba todas as atividades referentes ao gerenciamento das necessidades já captadas ao longo do processo de desenvolvimento do negócio. O modelo proposto por Rozenfeld (2006) propõe uma modelagem do produto funcional. De acordo com Hass e Wessel (2008), os modelos ou protótipos servem para clarificar as atividades da análise e garantir a validação funcional do que será entregue ao cliente.

Rozenfeld (2006) cita diretamente o monitoramento da viabilidade econômica do produto ou serviço estudado como uma etapa do processo de desenvolvimento de produtos, com foco na continuidade ou não do processo de desenvolvimento. Para Young (2003) e Babok (2011), este estudo da viabilidade faz parte do processo de definição de restrições e suposições, que analisa a viabilidade de se implementar a solução de acordo com as limitações da organização. Para um melhor planejamento dos desenvolvimentos, segundo Babok (2011) é importante que os requisitos possam ser separados em pacotes. Os pacotes devem ser preparados para uma avaliação antecipada da qualidade e planejamento do processo, de uma forma clara e compreensível das pequenas partes que irão compor o que será desenvolvido e entregue ao cliente.

O marco final da Fase 3 se dá pelo gerenciamento da rastreabilidade dos requisitos, citada separadamente por Babok (2011) como uma atividade específica, que entende que alterações no documento de requisitos são inevitáveis, seja pela identificação de erros ou pelo surgimento de novas necessidades do ponto de vista dos stakeholders. Para os demais autores, a rastreabilidade não é uma atividade e sim algo inerente ao processo, visto que um requisito para estar bem delimitado, precisa ser rastreável. Endende-se que um requisito é rastreável quando é possível determinar quem sugeriu o requisito, por que ele existe, a quais outros ele está relacionado e como ele está vinculado a outras informações do projeto. 


\subsubsection{Fase 4: Validação}

De acordo com o enfoque de melhoria contínua, ao final de cada etapa deve existir uma validação acerca do que foi efetuado. Dessa maneira, a Fase 4 é denominada como "Validação" e consiste em examinar todo o processo de forma a assegurar que os requisitos foram definidos sem ambiguidades, inconsistências ou omissões e que todos os erros foram detectados e resolvidos e que a solução atenda a todas as necessidades já declaradas. Young (2003) e Babok (2011) citam a etapa de testes e verificação antes da validação de requisitos, enquanto que para outros autores estas atividades não são citadas diretamente, mas fazem parte do processo.

Após os testes, os requisitos são validados e aprovados ou, quando necessário, passam por um novo processo de captação de requisitos, iniciando novamente o ciclo de gerenciamento de requisitos. Marx e Paula (2011) não cita diretamente atividades relacionadas a etapa de validação de requisitos, porém contempla algumas atividades já no processo de controle das mudanças que será detalhada na próxima etapa de monitoramento de requisitos. Por fim, esta etapa de validação dos requisitos é finalizada com a validação formal dos requisitos, já no formato da solução proposta com a área requisitante.

\subsubsection{Fase 5: Monitoramento de requisites}

Para manter uma lógica sequencial, a Fase 5, foi posicionada no fim do processo de gerenciamento de requisitos, porém, esta ocorre paralelamente durante todo o processo de desenvolvimento, embora seja mais mencionada no final. Tal fato ocorre por muitos modelos citados se basearem na Engenharia de Software, conceito que se fragmenta em Engenharia de Requisitos e GR, sendo a engenharia o processo como um todo e a gestão, o controle e monitoramento, alocados no final do processo.

No comparativo dos modelos, as atividades que compõem o monitoramento dos requisitos são as relacionadas ao gerenciamento das mudanças, monitoramento, planejamento e gerenciamento de requisitos. Tais atividades correspondem a um 
processo que serve de base para análise de indicadores e verificação da qualidade do processo de GR como um todo. Além disso, essa macro fase é fundamental para o controle do processo e tomada de ações com relação a melhorias na GR como um todo, especificamente nas etapas críticas que gerem retrabalhos em etapas subsequentes. Para cada tipo de produto esta etapa tem uma necessidade diferenciada, sendo mais importante no caso de inovações, em que as mudanças ao longo do processo são mais frequentes e devem ser realizadas até o final do projeto (MARX e PAULA, 2011).

\subsection{Apresentação das etapas consolidadas do modelo}

A análise comparativa realizada entre os diferentes modelos resultou em uma lista com atividades genéricas que abrangem todas as atividades identificadas nos diferentes modelos. O modelo integrado é apresentado na Figura 2, onde são detalhadas as etapas de cada fase, de acordo com o seu escopo e também são listadas as referências de onde cada atividade teve sua origem.

Para a definição dos nomes das fases foi utilizada a análise de conteúdo, a partir da regra de categorização, onde as atividades foram agrupadas de acordo com o seu principal objetivo, dando origem a cinco grandes fases no processo de Gestão de Requisitos - Elicitação, Especificação, Gerenciamento do Escopo, Validação e Monitoramento de Requisitos. A nomenclatura utilizada para a determinação das atividades foi baseada na repetição de cada modelo seguindo um determinado padrão. No caso dos modelos divergirem na atividade, utilizou-se basicamente a nomenclatura das atividades do modelo proposto por Babok (2011) visto que esse trabalho utiliza a perspectiva da análise de negócios, foco do presente estudo.

Além disso, para abranger o escopo da GR por completo, o Apêndice A apresenta um quadro contendo todas as ferramentas citadas para cada etapa do modelo integrado e os respectivos autores que as sugeriram. Esta relação poderá ser utilizada para orientar as empresas com relação a que métodos, técnicas e ferramentas podem ser utilizadas em suas atividades para otimizar o seu processo de gerenciamento de requisitos. 
Figura 2 - Proposta de um Modelo Integrado de Gestão de Requisitos

\section{Fase} Etapas

\begin{tabular}{|c|c|c|c|}
\hline \multirow{4}{*}{$\begin{array}{l}\text { Fase 1: } \\
\text { Elicitação }\end{array}$} & Mapear o cenário & $\begin{array}{l}\text { Entender o cenário no qual a necessidade foi detectada (sua origem, relacionamentos, necessidades, restrições). Neste momento é } \\
\text { fundamental entender o porquê de ela ainda não ter sido feita até este momento. }\end{array}$ & Rozenfeld et al (2006); Marx (2009); \\
\hline & Identificar os interessados & $\begin{array}{l}\text { Fazer a identificação de todos os interessados e influenciados pela necessidade detectada. Informar a estes o processo de elicitação que será } \\
\text { realizado posteriormente. }\end{array}$ & $\begin{array}{l}\text { Kotonya \& Sommerville (2000); Young (2003); Rozenfeld et } \\
\text { al (2006); Marx (2009); Babok (2011); }\end{array}$ \\
\hline & Realizar elicitação & Reunir todos os interessados para levantar as necessidades do que vai ser desenvolvido. & $\begin{array}{l}\text { Kotonya \& Sommerville (2000); Young (2003); Rozenfeld et } \\
\text { al (2006); Marx (2009); Babok (2011); }\end{array}$ \\
\hline & Documentar elicitação & Documentar todas as necessidades levantadas na elicitação que servirão de base para o desenvolvimento. & $\begin{array}{l}\text { Kotonya \& Sommerville (2000); Young (2003); Marx (2009); } \\
\text { Babok (2011) }\end{array}$ \\
\hline \multirow{4}{*}{$\begin{array}{l}\text { Fase 2: } \\
\text { Especificação }\end{array}$} & Analisar conflitos & $\begin{array}{l}\text { Alinhar os requisitos levantados entre todos os interessados para minimizar possíveis lacunas e desentendimentos entre o que cada um } \\
\text { solicitou. }\end{array}$ & Marx (2009) \\
\hline & Análise dos requisitos & $\begin{array}{l}\text { Garantir que todos os requisitos entreguem valor para o negócio, cumpram suas metas e objetivos e satisfaçam a necessidade das partes } \\
\text { interessada. Recomenda-se um briefing para reforçar o que ficou definido. }\end{array}$ & $\begin{array}{l}\text { Kotonya \& Sommerville (2000); Young (2003); Rozenfeld et } \\
\text { al (2006); Marx (2009); Babok (2011); }\end{array}$ \\
\hline & Priorização de requisitos aprovados & Determinar a importância relativa dos requisitos baseado nos critérios definido pelos interessados & $\begin{array}{l}\text { Kotonya \& Sommerville (2000); Young (2003); Marx (2009); } \\
\text { Babok (2011) }\end{array}$ \\
\hline & Especificar requisitos & Tradução dos requisitos em especificações, documentos técnicos e funcionais da solução que atenderá a necessidade. & $\begin{array}{l}\text { Kotonya \& Sommerville (2000); Young (2003); Rozenfeld et } \\
\text { al (2006); Marx (2009); Babok (2011); }\end{array}$ \\
\hline \multirow{4}{*}{$\begin{array}{l}\text { Fase 3: } \\
\text { Gerenciamento do } \\
\text { escopo }\end{array}$} & Modelar o produto & Fazer um protótipo simples do produto para avaliar as funcionalidades necessárias dentro da solução proposta. & Rozenfeld et al (2006); Marx (2009); \\
\hline & Definir suposições e restrições & $\begin{array}{l}\text { Definir todas as restrições e suposições que podem afetar a solução escohida para definir planos de ação que minimizem o seu impacto. } \\
\text { Suposições e restriç̃oes devem ser questionadas para garantir que a solução prposta seja válida, com um custo benefício aceitável. }\end{array}$ & Young (2003), Rozenfeld (2006), Marx (2009); Babok (2011) \\
\hline & Organizar e preparar pacote de requisitos & $\begin{array}{l}\text { Alocar os requisitos da solução em pacotes menores para estes serem desenvolvidos separadamente, minimizando a possibilidade de erros } \\
\text { que impactem de forma significativa o sistema. }\end{array}$ & Kotonya \& Sommerville (2000); Young (2003), Babok (2011) \\
\hline & Gerenciar rastreabilidade de requisitos & $\begin{array}{l}\text { Documentar todas as alteraç̃ões dos requisitos, explicitando quem sugeriu o requisito, porque ele existe, a quais outros ele está relacionado e } \\
\text { como ele está vinculado a outras informações do projeto. }\end{array}$ & Babok (2011) \\
\hline \multirow{2}{*}{$\begin{array}{c}\text { Fase 4: } \\
\text { Validação }\end{array}$} & Planejar testes de requisitos & $\begin{array}{l}\text { Com base nos requisitos, planejar os testes de funcionalidade da solução proposta, considerando tudo o que deve ser atendido, sua } \\
\text { performance, relacionamentos, restrições, etc. }\end{array}$ & $\begin{array}{l}\text { Kotonya \& Sommerville (2000); Young (2003); Rozenfeld et } \\
\text { al (2006); Marx (2009); Babok (2011); }\end{array}$ \\
\hline & Validar os requisitos & Validação do próprio produto já em funcionamento para avaliar se todas as necessidades foram atendidas. & $\begin{array}{l}\text { Kotonya \& Sommerville (2000); Young (2003); Rozenfeld et } \\
\text { al (2006); Marx (2009); Babok (2011) }\end{array}$ \\
\hline \multirow{2}{*}{$\begin{array}{l}\text { Fase 5: } \\
\text { Monitoramento }\end{array}$} & Controlar as mudanças nos requisitos & $\begin{array}{l}\text { Utilizar ferramentas para o controle das mudanças nos requisitos para poder gerenciar os artefatos relacionados a eles e também possíveis } \\
\text { indicadores. Seu objetivo é documentar os motivos da alteração visando o controle e análise do seu impacto com relação ao sistema. }\end{array}$ & Young (2003); Rozenfeld et al (2006) \\
\hline & Planejar o gerenciamento de requisitos & $\begin{array}{l}\text { Determinar o processo de gerenciamento de requisitos apropriado para cada caso em particular, desde as atividades de captação até a } \\
\text { entrega do produto. Isso incluir determinar o processo de evolução dos requisitos, quais partes precisam aprovar as possíveis mudanças, } \\
\text { quem deve ser consultado e informado e consequentemente, quem não deve. }\end{array}$ & Kotonya \& Sommerville (2000); Young (2006); Babok (2011) \\
\hline
\end{tabular}

Revista Produção Online, Florianópolis, SC, v.15, n. 4, p. 1481-1510, out./dez. 2015. 


\subsection{Diagnóstico de uma empresa utilizando o modelo proposto}

Com o objetivo de avaliar a viabilidade do modelo resultante nas etapas anteriores, este foi comparado com o processo realizado em uma empresa de Tecnologia da Informação com foco na gestão da cadeia de suprimentos e demanda, a qual promove soluções para integração dos negócios entre fornecedores, distribuidores, representantes, varejistas e clientes finais. O setor no qual o estudo foi conduzido é o de análise de negócios, responsável pelo processo de GR. Este setor participa e colabora com os projetos, visando a satisfação do cliente, adotando as melhores práticas de desenvolvimento de sistemas para avaliar as tecnologias disponíveis, captando os requisitos, elaborando documentos de especificação e homologação para detalhar de forma concreta quais são os objetivos do cliente.

Para a condução do estudo, foi utilizado o processo de triangulação de dados proposto por Yin (2001), aplicando-se três diferentes atividades: entrevistas, análise de documentos específicos e observação direta - para entender o processo de GR na empresa e compará-lo com o modelo proposto. Após isso, o resultado das análises foi compilado e foi possível a obtenção de um percentual médio de realização de modo que se pudesse diagnosticar as atividades mais críticas do processo, o que pode ser verificado na Figura 3.

As atividades realizadas pela empresa que ficaram com os níveis mais elevados de notas apesar de não terem atingido $100 \%$, foram justificadas pelo fato de a empresa não possuir uma metodologia padrão conhecida por todos, como é o caso das atividades de mapeamento do cenário, realização e documentação da elicitação, análise e especificação de requisitos.

As atividades classificadas com um nível intermediário (60 a 75\%) já tiveram outras causas que justificaram suas notas inferiores. A identificação dos interessados não possui um plano de comunicação para os envolvidos durante todo o processo, o que prejudica o alinhamento e gerenciamento das mudanças ao longo do processo. A priorização de requisitos é realizada por um comitê do produto, porém o fato de tal atividade ocorrer somente uma vez ao mês resulta em atrasos do processo. Além disso, os critérios para a priorização dos requisitos não são 
conhecidos por todos os envolvidos no processo. A atividade relativa a organizar e preparar o pacote de requisitos não ocorre de uma forma estruturada e é realizada somente em projetos maiores e não a nível de cada requisito que compõe o negócio analisado. Na validação de requisitos, em muitos casos se prioriza a validação das funcionalidades já existentes do que as propriamente desenvolvidas no momento da homologação, o que não corresponde ao objetivo principal da atividade. Por fim, o planejamento do GR, de uma forma geral deve ser estruturado, pois atualmente não é uma prática da empresa parar para estruturar o processo de cada nova necessidade encontrada.

Figura 3 - Análise comparativa no nível de atividades das diferenças entre o caso prático e real

Modelo Integrado de GR

Modelo utilizado na Empresa

\begin{tabular}{|l|l|c|}
\hline Mapear o cenário & Entender os requisitos iniciais & $89,2 \%$ \\
\hline Identificar os interessados & Agendar captação de requisitos & $72,5 \%$ \\
\hline Realizar elicitação & Planejar e realizar captação & $87,6 \%$ \\
\hline Documentar elicitação & Documentar requisitos levantados & $84,4 \%$ \\
\hline Analisar conflitos & - & $0,0 \%$ \\
\hline Análise dos requisitos & Validar requisitos com a área de negócio & $80,0 \%$ \\
\hline Priorização de requisitos aprovados & Validação do Comitê do Produto & $72,5 \%$ \\
\hline Especificar requisitos & Montar especificação & $91,3 \%$ \\
\hline Modelar o produto & Fazer mockups & $54,4 \%$ \\
\hline Definir suposições e restrições & & $0,0 \%$ \\
\hline Organizar e preparar pacote de requisitos & Roadmap de requisitos & $68,8 \%$ \\
\hline Gerenciar rastreabilidade de requisitos & Versionar documentos & $65,0 \%$ \\
\hline Planejar testes de requisitos & Planejar caoss de testes & $75,0 \%$ \\
\hline Validar os requisitos & Validar com a área técnica & $68,8 \%$ \\
\hline Controlar as mudanças nos requisitos & Avaliar ajustes e mudanças & $41,3 \%$ \\
\hline Planejar o gerenciamento de requisitos & Planejar o gerenciamento de requisitos & $65,0 \%$ \\
\hline
\end{tabular}

Dentre as atividades com uma classificação de realização crítica (inferiores a $60 \%$ ou até mesmo não realizadas) foram levantadas as atividades de gerenciar a rastreabilidade de requisitos, modelar o produto, definir suposições e restrições, análise de conflitos e planejar o gerenciamento de requisitos. A documentação utilizada hoje para os requisitos não possui rastreabilidade, sendo apenas possível se identificar qual a versão atual do documento, que tipo de alteração foi feita e por quem, não considerando o motivo pelo qual o requisito ter sido alterado nem a possível causa da falha. Além disso, as alterações normalmente são documentadas 
somente quando o desenvolvimento ainda está em andamento, depois que a melhoria ou novo desenvolvimento foi entregue, os documentos não continuam sendo realizados com a mesma frequência. A modelagem de produtos é feita pela empresa a nível de mockup, ou seja, de um desenho do que deve ser realizado, porém não é frequentemente utilizado um protótipo a nível funcional. $O$ nível funcional somente é realizado em casos críticos de processo, para otimizar o tempo que seria gasto em diversas outras etapas que não se teria tempo hábil de desempenhar. A atividade de controle de mudança nos requisitos ainda está em fase de construção na empresa. Algumas mudanças mais críticas são contabilizadas, mas poucos indicadores são gerados a partir delas.

Conforme apontado pelos entrevistados, a única das atividades considerada como crítica com a qual a empresa não pretende atuar em melhorias diretas é a de modelagem do produto, pois a criação de protótipos não é aplicável em todos os casos para a empresa, e o custo de sua realização não traria os benefícios que as justificassem. Além disso, o principal receio da empresa com relação às mudanças propostas em seu processo consiste no risco de burocratização do processo, pois tal fato pode prejudicar o andamento e cronograma de algumas atividades. Com este diagnóstico foi possível identificar as lacunas presentes no processo atual da empresa e, desta forma, indicar o caminho no qual a mesma deve seguir para aprimorar o seu GR de acordo com o modelo integrado proposto neste estudo.

\section{CONCLUSÕES}

Este trabalho apresentou uma proposta de um modelo que integra e consolida diferentes abordagens do processo de GR, de maneira a se ter uma visão completa que considere os detalhes das etapas que devem ser gerenciadas. Assim sendo, o modelo proposto contempla uma visão abrangente que permite sua aplicação em empresas de diferentes segmentos de mercado, contribuindo na obtenção de projetos melhor estruturados, minimizando os retrabalhos do processo. A análise realizada promove uma compreensão de que as abordagens existentes para a gestão de requisitos possuem tanto etapas comuns como também diferenças, além de destacar quais atividades são essenciais no processo de GR. Desta forma, o 
modelo proposto foi sustentado por cinco etapas principais - elicitação, especificação, gerenciamento do escopo da solução, validação e monitoramento que visam eliminar as lacunas já descritas na literatura, fornecendo às empresas uma descrição genérica e didática do que deveria ser considerado no processo de gestão dos requisitos do produto.

Com relação à aplicação prática apresentada neste artigo, o diagnóstico realizado com a empresa permitiu que esta, que considerava seu processo de GR como consolidado, percebesse que existem diversos pontos de melhoria, principalmente no que diz respeito a focar seus esforços, inicialmente, nas atividades consideradas como críticas de acordo com as notas geradas pelo questionário. Com base nos resultados obtidos, pode-se considerar que o modelo proposto mostrou-se conciso e aplicável dentro das expectativas do estudo visto que a empresa onde foi realizado o diagnóstico já está resultando em ações para a reestruturação de seu processo focando em indicadores de causas de retrabalhos. Além disso, um dos desafios deste trabalho é justamente a desmistificação de que controlar é burocratizar o processo já realizado. A implementação da metodologia não necessariamente precisa ser realizada de forma rígida conforme a literatura propõe, mas sim ajustada de acordo com a realidade da empresa.

\section{Limitações e propostas futuras}

O modelo proposto apresenta como limitação a base teórica utilizada para a sua construção que esteve baseada na literatura, e é possível que, com a utilização de outros modelos, novos resultados possam ser gerados. Neste caso, como trabalhos futuros, recomenda-se o aprimoramento da pesquisa na extensão para outras áreas com o intuito de expandir a sua aplicação e corroborar as proposições levantadas.

A aplicação prática, por ter baseado-se apenas em uma empresa, também possui limitações. Portanto, para futuras pesquisas seria importante desenvolver uma pesquisa survey com um conjunto maior de empresas, permitindo obter comparações dos resultados. Por fim, também seria apropriado o acompanhamento da estruturação do processo de gestão de requisitos de uma forma mais detalhada 
para que seja possível analisar o processo de mudança e mensurar a melhoria que tal estruturação pode trazer a empresa.

\section{Aplicações gerenciais}

Os resultados apresentados neste trabalho têm implicações relevantes para gestores e profissionais. Para a aplicação do modelo proposto, inicialmente, é preciso que o gestor entenda o seu processo e suas limitações, identificando suas atividades críticas. A partir deste ponto as atenções devem ser concentradas para a otimização dessas atividades de modo que o novo processo possa ser aprimorado, através do desenvolvimento e aprendizagem dos envolvidos, aplicando, na medida do possível, métodos e ferramentas já citados neste trabalho. Tais aplicações poderão, além de estruturar o gerenciamento de requisitos, facilitar o fluxo de informação do processo de modo que se atue nas causas dos problemas minimizando assim, possíveis retrabalhos para atingir os resultados da empresa. Por fim, esta gestão aplicada a requisitos deve considerar aspectos não só processuais, mas este também pode gerar resultados com impacto direto em outras áreas como a de planejamento de projetos, a contribuição para um ambiente de trabalho adequado, um fluxo de informação conciso e também auxílio à tomada de decisão.

\section{REFERÊNCIAS}

ARANTES, E.; ANSELMO, J.; SENSE, L.; SIBINELLI, P. Gerenciamento de projetos. Rio de Janeiro: Promom Business \& Technology, 2008.

BARDIN, L. Análise de Conteúdo. 3rd ed. Lisboa, 2004.

BEAL, A. Measuring the performance of business analysts. Disponível em: www.bealprojects.com. Acesso em: 05/03/2014.

BLASCHEK, José Roberto. Gerência de Requisitos: O principal problema dos projetos de Software. Developers CIO Magazine, Rio de Janeiro, n. 70, p. 46-47, 2002.

BOAVENTURA, E, M. Metodologia da pesquisa: monografia; dissertação; tese. São Paulo: Atlas, 2009.

BRAY, I. K. An introduction to requirements engineering. Pearson Education Limited. UK, 2002. 
BROOKS, F. P. No silver bullet. April, 1987.

CHARETTE, Robert N. Why software fails. IEEE spectrum, v. 42, n. 9, p. 36, 2005. DOI: http://dx.doi.org/10.1109/MSPEC.2005.1502528

CORDEIRO, Aline Gomes; FREITAS, André Luís Policani. Priorização de requisitos e avaliação da qualidade de software segundo a percepção dos usuários. Ciência da Informação, v. 40, n. 2, 2012. DOI: http://dx.doi.org/10760/17660.

FALBO, Ricardo Almeida. Integração de conhecimento em um ambiente de desenvolvimento de software. 1998. 205 p. 1998. Tese (Doutorado em Ciências em Engenharia de Sistemas e Computação) - Programa de Pós-Graduação de Engenharia, Universidade Federal do Rio de Janeiro, Rio de Janeiro.

GODOY, Arilda Schmidt et al. Introdução à pesquisa qualitativa e suas possibilidades. RAERevista de Administração de Empresas, v. 35, n. 2, 1995. DOI: http://dx.doi.org/10.1590/S0034-75901995000200008.

GRUHN, Volker. Process-centered software engineering environments, a brief history and future challenges. Annals of Software Engineering, v. 14, n. 1-4, p. 363-382, 2002. DOI: http://dx.doi.org/10.1023/A:1020522111961

HASS, Kathleen B.; WESSELS, Don J.; BRENNAN, Kevin. Getting it right: business requirement analysis tools and techniques. Management Concepts Inc., 2007.

HAZAN, Claudia; BERRY, Daniel M.; DO PRADO LEITE, Julio Cesar Sampaio. É possível substituir processos de Engenharia de Requisitos por Contagem de Pontos de Função?. In: WER. 2005. p. 197-208.

INTERNATIONAL INSTITUTE OF BUSINESS ANALYSIS (IIBA). Um Guia para o corpo de conhecimento de análise de negócios (Guia Babok). Canadá: International Institute of Business Analysis (IIBA), 2. Ed. 2011.

JIAO, J. R.; CHEN, C. H. Customer requirement management in product development: a review of research issues. Concurrent Engineering: Research and Applications, v. 14, n. 3, p. 173-185, set. 2006. DOI: http://dx.doi.org/10.1177/1063293X06068357

KAMARA, John M.; ANUMBA, Chimay J.; EVBUOMWAN, Nosa FO. Capturing client requirements in construction projects. London: Thomas Telford, 2002.

KAMARA, J. M.; ANUMBA, C. J.; EVBUOMWAN, N. F. O. Establishing and processing client requirements: key aspect of concurrent engineering in construction. Engineering

Construction and Architectural Management, v. 7, n. 1, p. 15-28, 2000. DOI: http://dx.doi.org/10.1108/eb021129

KOSCIANSKI, A.; SOARES, M. S. Qualidade de software. São Paulo: Novatec, 2006.

KOTONYA, G.; SOMMERVILLE, I. Requirements engineering: process and techniques. chichester: john wiley \& sons, 1998.

KOZLENKOV, Alexander; ZISMAN, Andrea. Are their design specifications consistent with our requirements?. In: Requirements Engineering, 2002. Proceedings. IEEE Joint 
International Conference on. IEEE, 2002. p. 145-154. DOI:

http://dx.doi.org/10.1109/ICRE.2002.1048517

LARKOWSKI, K. Latest Standish Group CHAOS report shows project success rates have Improved by 50\%. Standish Group International, West Yarmouth, MA, p. 1, 2003.

LEFFINGWELLI, D. WIDRING, D.; Managing Software Requirements: a unified approch, Addison-Wesley, 2000.

MARTINI, L.; CRUZ, J.; TRABASSO, L. G. Formalization of the requirements management process in the aerospace industry. Product: Management \& Development, 2003.

MARX, Â. PAULA, I. C de. Proposta de um método de engenharia de requisitos para o desenvolvimento de produtos sustentáveis. Production v. 21, n.3. São

Paulo jul./set. 2011 Epub 26-Ago-2011.

DOI: http://dx.doi.org/10.1590/S0103-65132011005000041

MYERS, G. J. The art of software testing, 1979.

NEVES, M. Análise de negócios para curiosos. 1. ed. São Paulo, 2012. Disponível em: www.giganteconsultoria.com.br/portal/index.php?option=com_lyftenbloggie\&view=entry\&yea $r=2012 \&$ month=04\&day=13\&id=30:videooqueean\&ltemid=9. Acesso em: 12/04/2014.

PARVIAINEN, P.; TIHINEN, M.; VAN SOLINGEN, R. Requirements engineering: dealing with the complexity of Sociotechnical Systems Development. In: MATÉ, J. L.; SILVA, A. Requirements engineering for sociothechnical systems. Hershey: Information Sciece Publiching, 2005. Cap. 2 DOI: http://dx.doi.org/10.4018/978-1-59140-506-1.

PODESWA, H. The business analyst handbook. 2nd ed. São Paulo, 2008.

PRESSMAN, R.S. Engenharia de software. São Paulo: Makron Books, 1995.

ROZENFELD, H. et al. Gestão de desenvolvimento de produtos: uma referência para a melhoria do processo. São Paulo: Saraiva, 2006.

SALLES, T. P. Melhorias no processo de comunicação e gerenciamento de requisitos alinhado ao BABOK: um estudo de caso. Revista de Sistemas e Computação, Salvador, v. 1, n. 2, p.120-138, jul/dez 2011.

SOMMERVILLE, I. Engenharia de Software. 8. ed. São Paulo: Ed. Addisson Wesley, 2007.

SUN, M.; SEXTON, M., AOUAD, G., FLEMING, A., SENATATNE, S., ANUMBA, C.

Managing changes in construction project. UWE: Bristol, 2005. Disponível em $<$ http://www.built-environment.uwe.ac.uk/research/cprc/publications/mcd.pdf >. Acesso em: 16/03/2014.

Trilha Análise de Negócio. Desenvolvido pela Globalcode Cursos de TI. São Paulo, 2013. Disponível em: <www.thedevelopersconference.com.br/tdc/2013/saopaulo/trilha-analise-deNegocio>. Acesso em: 12/04/2014.

TEMPLETON, G. Successfully managing business analysts. Message Consulting, 2012. Disponível em: www.messageconsulting.com/2012/05/successfully-managing-businessanalysts. Acesso em: 11/04/2014. 
THONG, James YL et al. Consumer acceptance of personal information and communication technology services. Engineering Management, IEEE Transactions on, v. 58, n. 4, p. 613625, 2011. DOI: http://dx.doi.org/10.1109/TEM.2010.2058851.

ULRICH, K. T.; EPPINGER, S. D. Product design and development. 2nd ed. New York: The McGraw-Hill Companies, 2000.

YIN, R. K. Estudo de caso: Planejamento E Métodos. Tradução de Daniel Grassi, 2. ed. Porto Alegre: Bookman, 2001.

YOUNG, R. The requirements engineering handbook. Norwood: Artech House, 2003.

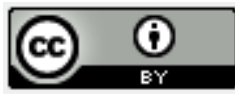

Artigo recebido em 03/04/2015 e aceito para publicação em 17/06/2015

DOI: http://dx.doi.org/ 10.14488/1676-1901.v15i4.2029 
APÊNDICE A - Ferramentas Sugeridas para o Processo de Gestão de Requisitos Quadro contendo Ferramentas Sugeridas na Literatura por etapa do Processo de Gestão de Requisitos Compilado. Fonte: Adaptado Marx e Paula (2011)

\begin{tabular}{|c|c|c|c|c|c|}
\hline $\begin{array}{c}\text { Ferramentas } \\
\text { Métodos } \\
\text { Técnicas }\end{array}$ & 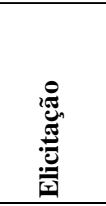 & 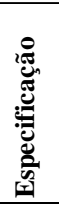 & 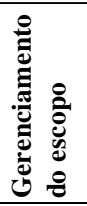 & 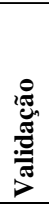 & 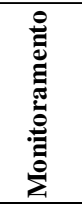 \\
\hline Abstração orientada & & & & 3 & \\
\hline Análise de documentos & 4 & & & & \\
\hline Análise de interface & 4 & & & & \\
\hline Análise de riscos & & & & 4 & \\
\hline Análise de regras & & 4 & 4 & & \\
\hline Análise etnográfica & 1 & & & & \\
\hline Análise matricial & & & 3 & & \\
\hline Análise paramétrica & & & 3 & & \\
\hline Associação com fases no ciclo de vida do produto & 3 & & & & \\
\hline Benchmarking & & & 3 & & \\
\hline Brainstorming & 3,4 & & & & \\
\hline Cenários e casos de uso & & & 4 & & \\
\hline Checklist & 3 & & 1 & 4 & \\
\hline Classificação em cliente (interno, externo) & 3 & & & & \\
\hline Decomposição funcional & & & 4 & & \\
\hline Definição dos crité rios de aceite & & & & 4 & \\
\hline Diagrama de afinidades & & 3,4 & & & \\
\hline Diagrama de Kano & 4 & & 3 & & \\
\hline Diagrama de Mudge & & & 3 & & \\
\hline Entre vis tas & $1,2,3,4$ & & & & \\
\hline Estruturas de desdobramento do ciclo de vida & 3 & & & & \\
\hline Gestão de requisitos (mudanças) & & & & & $1,2,3$ \\
\hline Grupos Focais & 4 & & & & \\
\hline Matriz de atributos & & & 3 & & \\
\hline Matriz de decisão & & & 4 & 3 & 4 \\
\hline Matriz de integrações & & & 1 & & \\
\hline Matriz de mapeamento & 3 & & & & \\
\hline Matriz de RACI & 4 & & & & 4 \\
\hline Métricas e indicadores chave de desempenho & & & & 4 & \\
\hline Modelagem funcional & & 4 & 4 & 3 & 4 \\
\hline Observação & 4 & & & & \\
\hline Pesquisa de mercado & 3 & & & & \\
\hline Prototipagem & $1,2,4$ & & & 4 & \\
\hline QFD & 3,4 & 3 & 3 & & \\
\hline Questionário estruturado & 3,4 & & & & \\
\hline Rastreamento de Problemas & & & & 4 & \\
\hline Reuniões & & & 1 & & \\
\hline Revisões estruturadas & & & & 4 & 4 \\
\hline Técnica de cenários & 1,2 & & & & \\
\hline Workshop de requisitos & 4 & & & & \\
\hline
\end{tabular}

[1] Kotonya e Sommerville (1998), [2] Young(2003), [3] Rozenfeld (2006), [4] Babok (2011) 